\title{
VIOLÊNCIA NO NAMORO: REFLEXÕES SOBRE A PROBLEMÁTICA NOS ADOLESCENTES
}

\author{
VIOLENCE IN DATING: REFLECTIONS ON A PROBLEM IN \\ ADOLESCENTS
}

Kalyne Araújo Bezerra ${ }^{1}$ Hirisdiane Bezerra Alves ${ }^{2}$ Pollyanna Jorge Canuto ${ }^{3}$

\begin{abstract}
RESUMO: OBJETIVO: Compreender sobre essa problemática da violência diante do namoro de adolescentes. MÉTODOS: Trata-se de uma revisão integrativa através da análise de estudos nas bases de dados BDENF, MEDLINE, LILACS, e IndexPsi Periódicos, através dos descritores "Violência" e "Adolescente" combinados pelo o uso do operador booleano "and", pesquisados na Biblioteca Virtual de Saúde BVS/BIREME. Foram encontrados 10 estudos relacionados, sendo 7 artigos, 1 dissertação, e 2 teses, e destes todos como pré-requisitos de serem textos completos, em português, e disponíveis online. Após refinamento, foram excluídos 4 , por não contemplarem os objetivos. Diante dos 6 artigos elencados para a análise, 0 ano de 2016 obteve o maior número de publicações, assim como a base LILACS também prevaleceu sobre a temática. RESULTADOS: Os estudos encontrados buscaram identificar as vulnerabilidades, traçar perfis de relacionamentos abusivos, compreensão acerca dos aspectos influenciadores, identificar características e perpetração deste agravo, e principalmente formas de intervir precocemente na perspectiva de diminuir as consequências. CONCLUSÃO: As reflexões oriundas do estudo endossam na intervenção relacionada a desnaturalização da violência, avaliar o quão precocemente as vítimas que estão em estado de vulnerabilidade e desmistificar a permissividade cultural existente na nossa sociedade, para também intervir no âmbito da prevenção e recuperação da saúde destes jovens.
\end{abstract}

Palavras chave: Violência; Adolescente; Corte.

ABSTRACT: OBJECTIVE: To understand about this problem of violence when dating teenagers. METHOD: This is an integrative review through analysis of studies in the

\footnotetext{
${ }^{1}$ Graduanda em Enfermagem na UNIFACISA.

${ }^{2}$ Graduada em Enfermagem pela UNINASSAU.

${ }^{3}$ Graduada em Enfermagem pela UNIFACISA, Mestranda em Saúde Pública pela UEPB.
} 
BDENF, MEDLINE, LILACS and IndexPsi Periodic databases, using the descriptors "Violence" and "Adolescent" combined by the use of the boolean operator "e", studied in the Library. Virtual Health - VHL / BIREME. We found 10 related studies, being 7 articles, 1 dissertation, 2 theses and all these full text prerequisites, in Portuguese, and available online. After refinement, 4 were excluded because they did not meet the objectives. Given the 6 articles listed for analysis, 2016 registered the largest number of publications, as well as the LILACS database also prevailed over the theme. RESULTS: The studies found seek to identify as vulnerabilities, track abuse records, understand about affected aspects, identify characteristics and perpetration of this problem, and especially early intervention in order to reduce the consequences. Conclusion: As reflections from the study endorsed in the intervention with denaturalization of violence, evaluate or calculate early how the lesions that are in the vulnerable state and demystify the cultural permissiveness existing in our society, also not to intervene in youth recovery and health.

KEYWORDS: Violence; Adolescent; Cut. 


\section{INTRODUÇÃO}

Segundo a OMS (2002), a violência consiste no uso da força física, ameaça e/ ou poder, de forma intencional contra si mesmo, contra o outro, contra um grupo de pessoas ou contra a comunidade, que possam gerar ou resultem em consequências psicológicas, físicas, deficiências no desenvolvimento e podendo resultar até mesmo em morte.

Sendo assim, a violência é considerada um grave problema de saúde pública, principalmente na fase da adolescência, pois trata-se do período em que os adolescentes se tornam mais vulneráveis, sobretudo à violência, que ocorre no próprio lar por seus pais, entre amigos, ou na comunidade (BRASIL, 2007). A partir da violência sofrida durante a infância, os adolescentes são possíveis perpetradores desse fenômeno, tornando-se agressores (BRASIL, 2018).

A adolescência é fase em que a sexualidade é assumida, surgindo os primeiros relacionamentos amorosos, podendo ser evidenciadas de diferentes maneiras a depender do meio e da aceitação em que está inserido (BRASIL, 2018). Com isso, é possível identificar o namoro, caracterizado pelo relacionamento em que existe compromisso entre moças e rapazes, e o "ficar", modalidade em que não há a responsabilidade com o outro (MINAYO, 2011).

Diante disso, aos adolescentes estarem em um relacionamento violento podem vivenciar a violência física, caracterizada pelo uso intencional da força para ferir ou matar; a emocional, que são os atos de ameaças e intimidação verbal que podem causar traumas emocionais; e sexual, que se refere ao envolvimento sexual sem o prévio consentimento, sendo exemplos a penetração e o contato sexual indesejado. A violência pode ocorrer por motivos de pressões e inseguranças, percepção errônea sobre o ciúme e inexperiência no namoro. Esse tipo de comportamento pode gerar danos a curto e longo prazo, como transtornos alimentares, estresse póstraumático, perturbações emocionais e comportamentos sexuais de risco (FREITAS, 2015). 
A violência no namoro é dividida em três fases. Na primeira fase, ocorre o aumento da tensão por meio de atitudes de intimidação e de controle por parte do agressor no dia a dia do casal, podendo resultar em discussões e posteriormente em agressões, entrando na segunda fase, em que ocorre o aumento de atos violentos e podem levar a morte. E o último estágio trata-se da reconciliação, na qual o agressor pede desculpas a vítima e muda o seu comportamento, oferecendo carinho, cuidado e atenção (FREITAS, 2015).

Desse modo, é de fundamental importância atuar principalmente na prevenção dos casos de violência no namoro por meio de conscientização educacional e comunitária, sendo de total relevância a participação dos pais nesse processo (RUBIO-GARAY et al., 2015).

Com isso, objetiva-se realizar uma síntese das produções científicas publicadas entre os anos de 2014 a 2019, acerca desta problemática e realizar reflexões sobre a mesma, de tal maneira que se consiga prospectar sobre a prevenção deste agravo a saúde dos adolescentes.

\section{METODOLOGIA}

Trata-se de um estudo de revisão integrativa, de natureza descritiva. Para isso, a busca foi realizada no mês de dezembro de 2019, na base de dados online Biblioteca Virtual em Saúde-BVS, com os descritores "Violência" e "Adolescente", juntamente com o termo "namoro" que não está registrado nos Descritores em Ciências da Saúde - DECS, combinados pelo o uso do operador booleano "and" que possuem texto completo, disponível em português, com recorte temporal nos últimos cinco anos (2014 a 2019). 


\section{RESULTADOS}

A pesquisa obteve como resultados 10 produções científicas, sendo composta por sete artigos, uma dissertação e duas teses, publicadas na BDENF - Banco de Dados em Enfermagem, LILACS - Literatura Latino Americana e do Caribe em Ciências da Saúde, MEDLINE - Medical Literature Analysis and Retrieval System Online e IndexPsi Periódicos-Index Psicologia Periódicos Técnicos-científicos. Porém apenas seis correspondiam ao objetivo proposto sendo excluídos os demais por não estarem totalmente relacionados a temática proposta neste estudo.

Durante a pesquisa pôde-se perceber que o ano de 2016 foi o que mais teve publicações relacionadas a temática da violência no namoro, em contrapartida, no ano de 2019 houve apenas uma publicação.

Dos documentos selecionados contam uma tese, uma dissertação e quarto artigos, publicados em 2019, 2016, 2015 e 2014.

\begin{tabular}{|c|c|c|c|c|c|c|c|}
\hline $\mathbf{N}^{\circ}$ & $\begin{array}{c}\text { Título da } \\
\text { publicação }\end{array}$ & Autor (s) & Objetivos & Ano & Tipo & $\begin{array}{c}\text { Base de } \\
\text { dados }\end{array}$ & Situação \\
\hline $\begin{array}{c}\text { Entre laços e } \\
\text { nós: narrativas } \\
\text { de violência nas } \\
\text { relações afetivo- } \\
\text { sexuais de } \\
\text { adolescente s de } \\
\text { uma escola na } \\
\text { região Costa } \\
\text { Verde (RJ) }\end{array}$ & $\begin{array}{c}\text { Carvalhaes, } \\
\text { R. de S. } \\
\text { as violências nas } \\
\text { relações afetivo- } \\
\text { sexuais são } \\
\text { significadas por } \\
\text { estudantes } \\
\text { adolescentes de uma } \\
\text { escola estadual da } \\
\text { região Costa Verde } \\
\text { do estado do Rio de } \\
\text { Janeiro }\end{array}$ & 2019 & Dissertação & LILACS & Disponível \\
\hline
\end{tabular}




\begin{tabular}{|c|c|c|c|c|c|c|c|}
\hline 2 & $\begin{array}{c}\text { Desafios éticos } \\
\text { na pesquisa com } \\
\text { adolescente s: } \\
\text { implicações da } \\
\text { exigência do } \\
\text { consentime nto } \\
\text { parental }\end{array}$ & $\begin{array}{l}\text { Borges, J. } \\
\text { L.; } \\
\text { Dell'Aglio, D. } \\
\text { D. }\end{array}$ & $\begin{array}{c}\text { Discutir os aspectos } \\
\text { éticos envolvidos na } \\
\text { pesquisa com } \\
\text { adolescentes, } \\
\text { incluindo a exigência } \\
\text { do termo de } \\
\text { consentimento dos } \\
\text { pais, da exigência do } \\
\text { consentime nto } \\
\text { parental }\end{array}$ & 2017 & Artigo & \begin{tabular}{|} 
LILACS e \\
Index \\
Psicologi \\
$a-$ \\
Periódico \\
s técnico- \\
científico \\
s
\end{tabular} & Disponível \\
\hline 3 & $\begin{array}{l}\text { Influência dos } \\
\text { Pares e } \\
\text { Educação por } \\
\text { Pares na } \\
\text { Prevenção à } \\
\text { Violência no } \\
\text { Namoro }\end{array}$ & $\begin{array}{l}\text { Santos, K. } \\
\text { B.; Murta, } \\
\text { S. G. }\end{array}$ & \begin{tabular}{|c|} 
Sumarizar aspectos \\
teóricos relativos à \\
influência dos pares \\
no contexto da \\
violência no namoro \\
edescrever estudos \\
com foco na \\
prevenção à \\
violência no namoro, \\
utilizadas no \\
desenvolvimen to \\
daintervenção \\
\end{tabular} & 2016 & Artigo & LILACS & Disponível \\
\hline 4 & \begin{tabular}{|c|} 
Violências \\
percebidas por \\
homens \\
adolescentes na \\
interação \\
afetivo- sexual \\
em dez cidades \\
brasileiras
\end{tabular} & \begin{tabular}{|l} 
Cecchetto, \\
F.; Oliveira, \\
Q. B.M.; \\
Njaine,K.; \\
Minayo, \\
M.C. de S.
\end{tabular} & $\begin{array}{c}\text { Apresentar as visões } \\
\text { de adolescentes do } \\
\text { sexo masculino sobre } \\
\text { o tema da violência } \\
\text { no namoro. }\end{array}$ & 2016 & Artigo & LILACS & Disponível \\
\hline 5 & $\begin{array}{l}\text { Efeitos de um } \\
\text { Programa de } \\
\text { Prevenção à } \\
\text { Violência no } \\
\text { Namoro }\end{array}$ & \begin{tabular}{|c|} 
Murta, S.G.; \\
Moore,R.; \\
Miranda,A. \\
A.V.; \\
Cangussú, \\
E.D.A.; \\
Santos,K.B \\
;Bezerra,K. \\
L.T.; Veras, \\
L.G. \\
\end{tabular} & $\begin{array}{c}\text { Avaliar os efeitos de } \\
\text { uma intervenção para } \\
\text { prevenção à violência } \\
\text { no namoro }\end{array}$ & 2016 & Artigo & $\begin{array}{c}\text { Index } \\
\text { Psicologi } \\
\text { a - } \\
\text { Periódico } \\
\text { s técnico- } \\
\text { científico } \\
\mathrm{s}\end{array}$ & Disponível \\
\hline 6 & $\mid \begin{array}{c}\text { Sexualidad e das } \\
\text { adolescente s em } \\
\text { situação de } \\
\text { acolhiment o: } \\
\text { contexto de } \\
\text { vulnerabilidade } \\
\text { para DST }\end{array}$ & \begin{tabular}{|c|} 
Penna, L. \\
H.G.; \\
Rodrigues, \\
R.F.; \\
Ribeiro, L. \\
V.; Paes, M. \\
V.; Guedes, \\
C. R.
\end{tabular} & $\begin{array}{c}\text { Analisar as situações } \\
\text { de vulnerabilidade na } \\
\text { saúde sexual de } \\
\text { adolescentes em } \\
\text { situação de } \\
\text { acolhimento. }\end{array}$ & 2015 & Artigo & $\left|\begin{array}{c}\text { BDENF e } \\
\text { LILACS }\end{array}\right|$ & Disponível \\
\hline
\end{tabular}




\begin{tabular}{|c|c|c|c|c|c|c|c|}
\hline 7 & \begin{tabular}{|c|} 
Violência nas \\
relações de \\
namoro de \\
adolescente s em \\
situação de \\
acolhiment o \\
instituciona l: um \\
olhar da \\
enfermage m
\end{tabular} & Lapa, A.T. & \begin{tabular}{|c|} 
Identificar as \\
características das \\
relações de namoro \\
das adolescentes em \\
situação de \\
acolhimento, bem \\
como analisar as \\
vivências e as \\
repercussões de \\
violência no namoro \\
analisar as vivências \\
da violência nas \\
relações de namoro \\
\end{tabular} & 2015 & Tese & BDENF & $\begin{array}{c}\text { Não } \\
\text { disponível }\end{array}$ \\
\hline 8 & \begin{tabular}{|c|} 
Namoro na \\
adolescênci a no \\
Brasil: \\
circularidad e da \\
violência \\
psicológica nos \\
diferentes \\
contextos \\
relacionais.
\end{tabular} & $\begin{array}{c}\text { Oliveira, Q. } \\
\text { B. M.; de } \\
\text { Assis, S. G.; } \\
\text { Njaine,K.; } \\
\text { Pires,T. O. }\end{array}$ & \begin{tabular}{|c|} 
Avaliar a \\
perpetração de \\
violência psicológica \\
no relacionamento \\
afetivo-sexual atual \\
de adolescentes e \\
sua relação com \\
violência psicológica \\
vivenciada na família, \\
em amizades e com \\
parceirosafetivo- \\
sexuais anteriores \\
\end{tabular} & 2014 & Artigo & $\begin{array}{c}\text { MEDLIN } \\
\mathrm{E}\end{array}$ & Duplicado \\
\hline 9 & \begin{tabular}{|c|} 
Violência de \\
gênero no \\
namoro entre \\
adolescente s \\
sob a ótica dos \\
adolescentes, \\
educadores e \\
profissionai s da \\
saúde
\end{tabular} & $\begin{array}{c}\text { Oliveira, Q. } \\
\text { B. M. }\end{array}$ & \begin{tabular}{|} 
Analisar a violência \\
no namoro entre \\
adolescentes e as \\
possibilidades de \\
enfrentamento desse \\
problema sob o \\
enfoque de gênero.
\end{tabular} & 2014 & Tese & LILACS & Disponível \\
\hline
\end{tabular}

\section{DISCUSSÃO}

\section{Caracterização da violência}

A prevalência de violência entre namorados é alta no Brasil e no mundo, sendo niveladas por valores semelhantes para $24 \%$ no Canadá, $24,3 \%$ na Espanha e $24,1 \%$ no Brasil. É ocasionada por ambos os sexos, sendo principalmente nas formas física e psicológica e também a violência verbal, que é recorrida pelos meninos como 
recurso para a não realização da violência física. De acordo com uma pesquisa realizada em dez capitais brasileiras, a violência física é realizada principalmente pelo menino, mas não descarta a realização da mesma pela menina, porém esta é banalizada e tida como forma de humilhação para os meninos (OLIVEIRA, 2014).

De acordo com os adolescentes, o comportamento de agressão no namoro é justificável por atitudes de ciúmes, infidelidade e revide a violência (CARVALHAES, 2019).

Sendo assim, é comum que os adolescentes procurem ajuda em situações de emergência no namoro em sua rede de amizade, que costumeiramente é composta por outros adolescentes que não possuem o devido conhecimento de como agir nessas situações e muitas vezes influenciem no relacionamento de forma negativa, introduzindo ou até mesmo agravando, a violência no namoro (SANTOS; MURTA, 2016).

\section{Tipos de violência}

Foram evidenciados seis tipos de violências, sendo elas: a verbal que é caracterizada por xingamentos (CECCHETTO et al., 2016); a física, que está presente no ato de jogar algo sobre o outro, nos chutes, batidas, empurrões, sacudidas, puxões de cabelo, socos e apertos no pescoço (CARVALHAES, 2019); a moral, por meio de chantagem, difamação, humilhação (CECCHETTO et al., 2016); a psicológica, quando é imposto o medo, o controle de amizade, roupas e ações do outro; a sexual, por beijos forçados, tocar sexualmente, forçar o sexo, ameaças para a prática de relações sexuais e sexo sem preservativo; a cyber violência também conhecida como abuso digital, ocorrida por ameaças e insultos via redes sociais e digitais, divulgação de fotos e vídeos íntimos sem autorização, controle de postagens e monitoramento de conversas pelo WhatsApp, Facebook e Instagram que ocorre por motivos de controle para as meninas e como vingança para os meninos após o término do relacionamento (CARVALHAES, 2019). 
A tipologia que se mostrou como a mais representativa, de acordo com o estudo realizado por Oliveira (2014) foi a violência física, perpetrada tanto pelo sexo feminino quanto pelo masculino, em que para os meninos, caracteriza-se como um símbolo de masculinidade. Enquanto que, na mesma pesquisa, porém na ótica dos profissionais de saúde e de educação baseada em suas vivências diárias, a violência mais predominante seja a verbal, sendo as meninas as mais agressivas nesta modalidade (OLIVEIRA, 2014).

\section{Fatores de risco}

Estudo mostra que, quanto maior o tempo de relacionamento, a probabilidade de ocorrência de violência no namoro entre adolescentes aumenta, bem como a idade, ou seja, quanto mais novos os namorados forem, maior o risco de violência. $A$ raça, escolaridade, condições socioeconômicas, renda familiar, violência intrafamiliar, baixa autoestima, grau de envolvimento na relação, isolamento social e tolerância a violência também são fatores que interferem na existência da violência (CARVALHAES, 2019).

De acordo com uma pesquisa realizada com 3.205 adolescentes de escolas públicas estaduais e particulares de diferentes estados brasileiros, a possibilidade da ocorrência de violência psicológica no relacionamento aumenta consideravelmente quando o adolescente tem sofrido algum tipo de violência na infância, podendo ela ser pelo pai, mãe, irmãos, amigos ou em relacionamentos anteriores (OLIVEIRA, et al., 2014).

\section{Fatores desencadeantes}

Segundo (CARVALHAES, 2019), as maiores causas são a vontade de controlar o outro, a traição, o término do relacionamento, a dificuldade em resolver 
conflitos e lidar com aspróprias emoções, uso e abuso de álcool e outras drogas e os ciúmes, que mostrou-se como sendo o maior causador de brigas entre os adolescentes namorados (CECCHETTO et al., 2016).

\section{Consequencias causadas pela violência no namoro}

Após a violência, os adolescentes podem sofrer danos físicos e emocionais, como por exemplo o estresse pós-traumático, baixa autoestima, raiva, depressão, ideação suicida, baixo rendimento escolar, sentimento de desvalorização (CARVALHAES, 2019), ansiedade, dores crônicas, efeitos danosos a saúde mental e na qualidade de vida, bem como pode desenvolver multiplicadores de violência entre casais na vida adulta (OLIVEIRA, 2014).

\section{Prevenção a violência}

Como ações de prevenção a violência, é notório a escassez dessas ações, principalmente no que tange aos profissionais da área da educação e de saúde. $\mathrm{Na}$ área da educação, a enorme quantidade de tarefas e atitude dos pais em tornar a escola como principal responsável pelos seus filhos, dificulta a prevenção. Enquanto que para os profissionais de saúde, as ações de prevenção a essa temática aumentaram sob efeito da Política Nacional de Redução da Morbimortalidade por Acidentes e Violências, com isso, estes profissionais tornaram-se conscientes da necessidade de realização de atividades educativas tendo como alvo os adolescentes e a capacitação de profissionais de outras áreas como os de educação (OLIVEIRA, 2014).

De acordo com o estudo realizado por Murta (2016), em que foi realizado uma intervenção de prevenção a violência com 45 adolescentes compreendendo a idade 
entre 15 e 17 anos, evidenciou que para os perpetradores houve uma diminuição na resposta agressiva e as vítimas, uma resposta mais assertiva. A partir de habilidades ensinadas aos adolescentes, houveram benefícios com o autocontrole com relação a emoções e sentimentos; a empatia, ou seja, os adolescentes passaram a pensar antes de tomar atitudes que pudessem magoar o namorado (a) e o diálogo no relacionamento.

\section{CONCLUSÃO}

Mediante a pesquisa, pode-se observar a necessidade de mais estudos com esta temática, enfatizando a carência no Brasil. A violência é uma realidade que está presente em diversos contextos do nosso cotidiano e em diferentes faixas etárias, tornando-se um fenômeno abrangente e de grande relevância.

$\mathrm{Na}$ adolescência torna-se mais agravante, pelo fato de ser a idade em que ocorrem mudanças no corpo e no comportamento fazendo com que o adolescente esteja vulnerável a diversos perigos, o que aumenta as ocorrências de violência.

Os estudos mostraram que, a violência é um assunto pouco discutido em diversos âmbitos, inclusive com a população adolescente e que apesar da existência de políticas públicas, as ações educativas e intervenções sobre a temática são insuficientes para promover uma conscientização do público, impedindo assim uma melhor qualidade de vida e promoção da saúde.

O público que está em contato direto com os adolescentes, deve estar consciente das atitudes a serem tomadas nestes casos, enfatizando principalmente os profissionais da saúde, que são de extrema importância e devem atuar tanto na prevenção quanto nos danos ocasionados pela violência, bem como realizar a notificação compulsória em casos suspeitos e em casos confirmados para que, os órgãos públicos possam ter ciência da prevalência e agir adequadamente de acordo com os dados. 


\section{REFERÊNCIAS BIBLIOGRÁFICAS}

BRASIL. Ministério da Saúde. Secretaria de Atenção à Saúde. Área de Saúde do Adolescente e do Jovem. Marco legal: saúde, um direito de adolescentes. Ministério da Saúde, Secretaria de Atenção à Saúde, Área de Saúde do Adolescente e do Jovem. Brasília: Editora do Ministério da Saúde,2007;

BRASIL. Ministério da Saúde. Secretaria de Atenção à Saúde. Departamento de Ações Programáticas e Estratégicas. Proteger e cuidar da saúde de adolescentes na atenção básica. Ministério da Saúde, Secretaria de Atenção à Saúde, Departamento de Ações Programáticas e Estratégicas. 2. ed. Brasília: Ministério da Saúde, 2018;

CARVALHAES, Renata de Souza. Entre laços e nós: narrativas de violência nas relações afetivo- sexuais de adolescentes de uma escola na região Costa Verde (RJ). 2019. 143 f. Dissertação (Mestrado) - Curso de Saúde Coletiva, Universidade do Estado do Rio de Janeiro, Rio de Janeiro, 2019;

CECCHETTO, Fátima et al. Violências percebidas por homens adolescentes na interação afetivo-sexual em dez cidades brasileiras. Interface - Comunicação, Saúde, Educação, [s.l.], v. 20, n. 59, p.853-864, 28 jun. 2016. FapUNIFESP (SciELO);

FREITAS, Carmina Patrícia Flores. A submissão associada à violência no namoro: um contributo para a promoção de competências sociais e emocionais. 2015. 177 f. Dissertação (Mestrado) - Curso de Psicologia, Universidade dos Açores, Ponta Delgada, 2015;

MINAYO, Maria Cecília de Souza; ASSIS, Simone Gonçalves de; NJAINE, Kathie (Org.). Amor e violência: um paradoxo das relações de namoro e do 'ficar' entre jovens brasileiros. Rio de Janeiro: Editora Fiocruz, 2011;

MURTA, Sheila Giardini et al. Efeitos de um Programa de Prevenção à Violência no Namoro. Psico-usf, [s.l.], v. 21, n. 2, p.381-393, ago. 2016. FapUNIFESP(SciELO);

OLIVEIRA, Queiti Batista Moreira. Violência de gênero no namoro entre adolescentes sob a ótica dos adolescentes, educadores e profissionais da saúde. 2014. $167 \mathrm{f}$. Tese (Doutorado) - Curso de Saúde Coletiva, Escola Nacional de Saúde Pública Sergio Arouca, Rio de Janeiro, 2014;

OLIVEIRA, Queiti Batista Moreira et al. Namoro na adolescência no Brasil: circularidade da violência psicológica nos diferentes contextos relacionais. Ciência \& Saúde Coletiva, [s.I.], v. 19, n. 3, p.707-718, mar. 2014. FapUNIFESP (SciELO);

RUBIO-GARAY, Fernando et al. Factores asociados a la violencia en el noviazgo entre adolescentes: una revisión crítica. Anuario de Psicología Jurídica, [s.I.], v. 25, n. 1, p.47-56, jan. 2015. Colégio Oficial de Psicólogos de Madrid;

SANTOS, Karine Brito dos; MURTA, Sheila Giardini. Influência dos Pares e Educação por Pares na Prevenção à Violência no Namoro. Psicologia: Ciência e Profissão, [s.l.], v. 36, n. 4, p.787-800, dez. 2016. FapUNIFESP (SciELO). 